\title{
A Importância da avaliação motora em escolares: análise da confiabilidade da Escala de Desenvolvimento Motor
}

Importance of motor assessment in school children: analysis of the reliability of the Motor Development Scale

Francisco Rosa Neto Ana Paula Maurilia Dos Santos Regina Ferrazoli Camargo Xavier Kassandra Nunes Amaro ${ }^{1}$

1 Universidade do Estado de Santa Catarina, Centro de Ciências da Saúde e do Esporte, Laboratório de Desenvolvimento Humano. Florianópolis, SC. Brasil.

Recebido em 27/10/09 Revisado em 08/02/10 Aprovado em 21/04/10

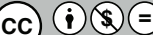

Resumo - O objetivo deste estudo foi: (1) investigar o perfil motor de escolares de 6 a 10 anos ( $n=101)$, sem queixas de dificuldades na aprendizagem; (2) analisar a confiabilidade da Escala de Desenvolvimento Motor-EDM (Rosa Neto, 2002). A análise descritiva dos dados foi feita através da média, desvio padrão, valor mínimo e valor máximo. A Consistência Interna da EDM foi analisada através do teste estatístico Alpha de Cronbach, e a correlação das variáveis pela Correlação Linear de Pearson, com valor de $p<0,05$. Os resultados sugerem: (1) desenvolvimento motor dentro dos parâmetros de normalidade em $96 \%$ dos escolares; (2) alta correlação entre a Idade Cronológica e Idade Motora Geral indicando boa consistência interna. Estes dados evidenciaram a concepção lógica e estruturada que a EDM apresenta, legitimando sua confiabilidade.

Palavras-chave: Escolares; Desenvolvimento motor; Consistência interna; Coeficiente alpha.

Abstract - The objective of this study was to investigate the motor performance of school children aged 6 to 10 years without learning difficulties $(n=101)$, and to analyze the reliability of the Motor Development Scale (MDS) (Rosa Neto, 2002). Descriptive statistics with calculation of the mean, standard deviation, and range was used for data analysis. The internal consistency of the MDS was assessed using Cronbach's alph a coefficient, and the correlation between variables was determined by Pearson's linear correlation, with $p<0.05$. The results showed (1) that motor development was within normal limits in 96\% of the children, and (2) a high correlation between chronological age and general motor age, indicating good internal consistency. These data demonstrate the logic and structured design of the MDS, confirming its reliability.

Key words: School children; Motor development; Internal consistency; Alpha coefficient. 


\section{INTRODUÇÃO}

Dentre as razões que têm levado ao interesse crescente pelos conhecimentos acerca do desenvolvimento motor, destacam-se os paralelos existentes entre o desenvolvimento motor e o desenvolvimento cognitivo. Há uma estreita relação entre o que a criança é capaz de aprender (cognitivo) com o que é capaz de realizar (motor).

O desenvolvimento motor é um processo sequencial, relacionado à idade cronológica, trazido pela interação entre os requisitos das tarefas, a biologia do indivíduo e as condições ambientais, sendo inerente às mudanças sociais, intelectuais e emocionais ${ }^{1}$. É na infância, particularmente, no início do processo de escolarização, que ocorre um amplo incremento das habilidades motoras, que possibilita à criança um amplo domínio do seu corpo em diferentes atividades, como: saltar, correr, rastejar, chutar uma bola, arremessar um arco, equilibrar-se num pé só, escrever, entre outras ${ }^{2}$

Além disso, a aquisição de habilidades motoras está vinculada ao desenvolvimento da percepção do corpo, espaço e tempo, e essas habilidades constituem componentes de domínio básico tanto para a aprendizagem motora quanto para as atividades de formação escolar ${ }^{3}$. Isso significa que, ao conquistar um bom controle motor, a criança estará construindo as noções básicas para o seu desenvolvimento intelectual. Por isso, o fato de se proporcionar o maior número de experiências motoras e psicossociais às crianças, estará se prevenindo que estas apresentem comprometimento de habilidades escolares ${ }^{4}$.

No contexto escolar, a prática da educação motora tem influência no desenvolvimento de crianças com dificuldades escolares, como problema de atenção, leitura, escrita, cálculo e socialização ${ }^{5}$. O que leva a considerar que o acompanhamento da aptidão motora de crianças em idade escolar constitui atitude preventiva para profissionais envolvidos com a aprendizagem.

Dentro desta concepção, a avaliação motora deve ser rotina nas escolas, possibilitando um melhor diagnóstico da criança, com um conhecimento mais aprofundado de suas possibilidades e limitações reais. O tema "avaliação" tem sido objeto de várias investigações nas áreas voltadas à Educação, em geral. Na área de Educação Física, o foco de discussões se relaciona à necessidade de oferecer um cunho profissional, sistemático, não-empírico e estruturado à tomada de decisões sobre o ensino ${ }^{6}$.

De acordo com os Parâmetros Curriculares Nacionais ${ }^{7}$ a avaliação na área da Educação Física se resume a testes de força, resistência e flexibilidade, medindo apenas a aptidão física do aluno. Desse modo, a aptidão motora não vem recebendo o devido acompanhamento, e as crianças que apresentam dificuldades nas realizações dessas tarefas, sob a perspectiva do senso comum, podem ser frequentemente chamadas de 'descoordenadas' ou 'desajeitadas'.

Existem inúmeros testes e escalas para avaliação do desenvolvimento motor de uma criança ${ }^{8}$, no entanto, quase nenhum desses instrumentos consegue englobar completamente todos os aspectos do desenvolvimento. Uma das escalas que tende a colaborar para a avaliação completa e elucidativa do desenvolvimento motor, é a Escala de Desenvolvimento Motor ${ }^{9}$. Esta escala, que possui um método de aplicação de testes atrativo para a criança, compreende um conjunto de provas diversificadas e de dificuldade graduada, abrangendo diferentes áreas do desenvolvimento motor.

Mais de 130 pesquisas científicas no Brasil, com diferentes tipos de população, já foram realizadas com a utilização deste instrumento, sendo que grande parte dos pesquisadores investigam crianças atípicas, com indicadores de algum tipo de distúrbio e escolares com dificuldades na aprendizagem, que por decorrência dessas desordens apresentam um desenvolvimento mais tardio nas funções motoras e também cognitivas.

Num outro paradigma, em crianças sem dificuldades na aprendizagem (com bom desempenho escolar), não significa que o desenvolvimento motor se constitui numa uniformidade a predizer bom desempenho e potencialidade, isto seria negar os conceitos de continuidade, individualidade no processo desenvolvimentista ${ }^{1}$. Porém, na hipótese de se dizer, se o nível de desenvolvimento motor desses escolares (típicos) obedece a uma sequência linear à sua idade cronológica, somente é possível através da avaliação motora.

Nessa perspectiva, o objetivo deste estudo foi traçar um perfil motor de crianças sem queixa de dificuldades na aprendizagem, apresentando o case do desempenho escolar e o desempenho motor. Além disso, este estudo propôs analisar a confiabilidade da referida Escala de Desenvolvimento Motor -EDM com o intuito de divulgar aos profissionais da área da saúde e educação um instrumento de diagnóstico eficaz.

\section{PROCEDIMENTOS METODOLÓGICOS}

O estudo foi aprovado pelo Comitê de Ética em Pesquisa em Seres Humanos, da Universidade do Estado de Santa Catarina (UDESC), com protocolo número 101/2008. 
A população foi composta por escolares de 6 a 10 anos, do $1^{\circ}$ ao $4^{\circ}$ ano do Ensino Fundamental de duas escolas públicas da Cidade de Florianópolis - SC. A amostra foi constituída por 101 escolares, de ambos os sexos (56 do sexo masculino e 45 do sexo feminino), com média de idade de 8,15 anos (97,91 meses). A seleção da amostra foi do tipo intencional, com o propósito de avaliar somente as crianças que não fossem repetentes, que não apresentassem dificuldades na aprendizagem, deficiências físicas, déficits sensoriais, transtornos comportamentais ou deficiência mental, além de ter, por escrito, autorização prévia dos pais ou responsáveis para participar do estudo.

A partir dessa característica amostral, pôde-se correlacionar a idade cronológica com a idade motora dos escolares, no intuito de conferir o grau de linearidade dessas variáveis e, do mesmo modo, analisar a confiabilidade do instrumento proposto.

$O$ instrumento utilizado para avaliar o desenvolvimento motor dos escolares foi o Protocolo de testes da Escala de Desenvolvimento Motor - $\mathrm{EDM}^{9}$, que avalia as seguintes áreas do desenvolvimento: motricidade fina (IM1), motricidade global (IM2), equilíbrio (IM3), esquema corporal (IM4), organização espacial (IM5), organização temporal (IM6) e lateralidade. Este instrumento determina a idade motora (obtida através dos pontos alcançados nos testes) e o quociente motor (obtido pela divisão entre a idade cronológica multiplicado por 100). Com exceção dos testes de lateralidade, as outras baterias consistem em 10 tarefas motoras cada, distribuídas entre 2 e 11 anos, organizadas progressivamente em grau de complexidade, sendo atribuído para cada tarefa, em caso de êxito, um valor correspondente a idade motora (IM), expressa em meses. Em cada bateria, o teste é interrompido quando a criança não concluir a tarefa com êxito, conforme protocolo. Ao final da aplicação, depen- dendo do desempenho individual em cada bateria, é atribuída à criança uma determinada IM, em cada uma das áreas referidas anteriormente (IM1, IM2, IM3, IM4, IM5, IM6), sendo após, calculada a idade motora geral (IMG) e o quociente motor geral (QMG) da criança. Esses valores são quantificados e categorizados, permitindo classificar as habilidades analisadas em padrões: muito superior (130 ou mais), superior (120-129), normal alto (110119), normal médio (90-109), normal baixo (80-89), inferior (70-79) e muito inferior (69 ou menos).

A EDM foi aplicada integralmente por dois avaliadores previamente treinados (profissionais de Educação Física e Fisioterapia), colaboradores do Laboratório de Desenvolvimento Humano do CEFID/UDESC. O tempo médio de aplicação dos testes foi de 35 minutos. A coleta de dados estendeu-se por um período de 3 meses.

Para o tratamento descritivo dos dados, foi utilizada a análise das médias, desvio padrão, valor mínimo e valor máximo. Na análise inferencial dos dados, foi realizado o Alpha de Cronbach, para examinar a Consistência Interna da Escala de Desenvolvimento Motor - EDM. Para verificar a correlação entre a variável Idade Cronológica e Idade Motora Geral, utilizou-se a Correlação Linear de Pearson. O nível de significância adotado para todas as análises foi de $\mathrm{p}<0,05$.

\section{RESULTADOS}

De acordo com os objetivos propostos pela pesquisa, de traçar um perfil motor de escolares e analisar a confiabilidade da Escala de Desenvolvimento Motor - EDM, os resultados serão apresentados em dois momentos: a) Perfil Motor do Grupo. b) Consistência Interna e Correlação Linear da EDM.

Perfil Motor do grupo

Tabela 1. Perfil Motor geral do grupo.

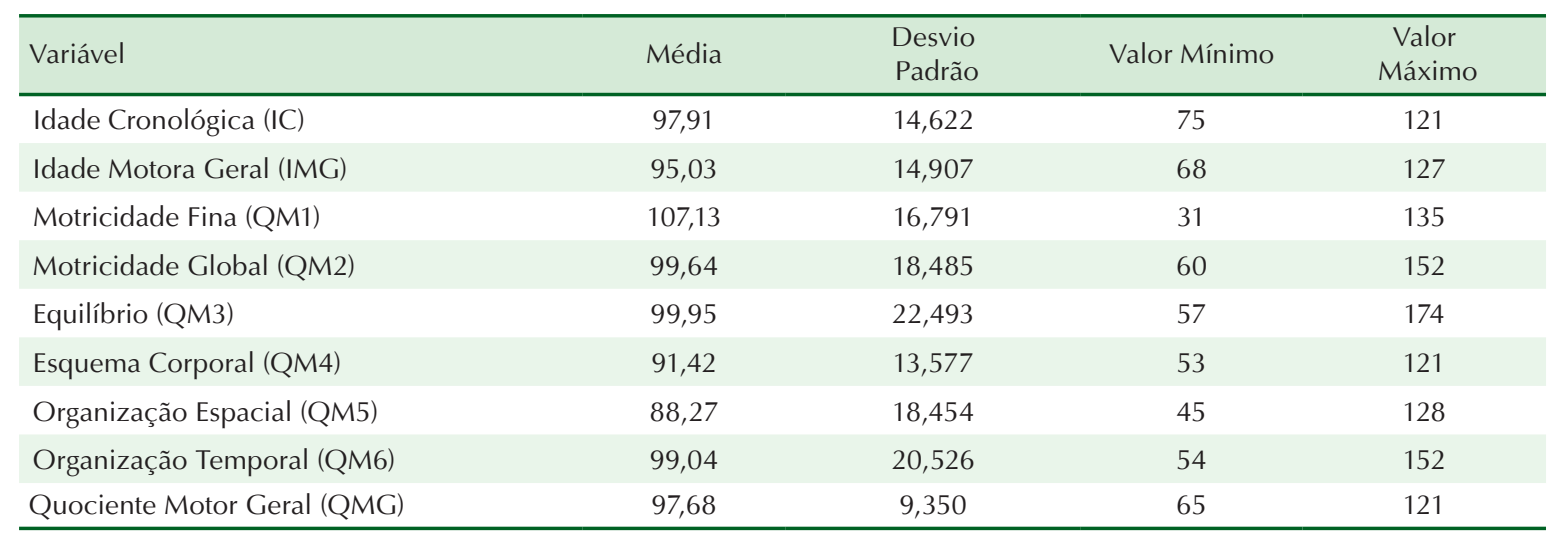

Nota: As idades são expressas em meses. 
Em relação às características motoras dos escolares, descritas na tabela I, verifica-se que a idade cronológica média da amostra foi de 97,91 meses, e a idade motora geral de 95,03 meses. Especificamente, todas as áreas motoras avaliadas obtiveram classificação normal médio (QM entre 90 a 109), exceto a organização espacial que foi categorizada como normal baixo (QM entre 80 a 89).

Percentualmente, na classificação geral dos escolares, 96\% ( $\mathrm{n}=97)$ deles apresentaram índices de desenvolvimento motor normal (normal alto, médio e baixo). Uma pequena discrepância foi caracterizada por $4 \%$ dos escolares, os quais, $1 \%(n=1)$ apresentou desenvolvimento motor acima da normalidade, e 3\% $(\mathrm{n}=3)$ abaixo da normalidade (Tabela 2).

Tabela 2. Classificação geral dos resultados da EDM

\begin{tabular}{lcc}
\hline Classificação & Freqüência & $\%$ \\
\hline Superior & 1 & 1,0 \\
Normal Alto & 11 & 10,9 \\
Normal Médio & 74 & 73,3 \\
Normal Baixo & 12 & 11,9 \\
Inferior & 2 & 2,0 \\
Muito inferior & 1 & 1,0 \\
Total & 101 & 100,0 \\
\hline
\end{tabular}

Na avaliação da lateralidade, evidenciou-se que 97\% dos escolares possuem lateralidade definida, no entanto, com preferência lateral variada. Destes, 59\% apresentaram preferência lateral direita - caracterizado como destro-completo (mãos, olhos, pés); $4 \%$ apresentaram preferência lateral esquerda (sinistro-completo) e 35\%, apresentaram lateralidade cruzada (escrevem com a mão esquerda e chutam com o pé direito, por exemplo). A lateralidade foi indefinida em $3 \%$ dos escolares.

Consistência Interna e Correlação Linear da EDM Quanto o grau de confiabilidade da bateria de testes da Escala de Desenvolvimento Motor - EDM, os dados motores da atual pesquisa, sendo submetidos ao teste de correlação linear de Pearson, demonstraram haver alta correlação $(0,800)$ entre as variáveis Idade Cronológica (IC) e Idade Motora Geral (IMG), indicando consistência interna boa $(0,889)$.

\section{DISCUSSÃO}

Os resultados deste estudo dão suporte às predições dos paralelos existente entre o desenvolvimento motor e cognitivo. O desenvolvimento motor dessas crianças que não apresentam queixa de di- ficuldades na aprendizagem foi situado, quase que na sua totalidade (96\%), dentro dos parâmetros da normalidade. Este achado corrobora outros estudos que relacionam desenvolvimento motor e rendimento escolar os quais demonstram significância estatística entre o que a criança é capaz de aprender (cognitivo) e o que é capaz de fazer (motor) ${ }^{4,10-14 .}$

Desse modo, o padrão de desenvolvimento motor encontrado na atual pesquisa, sob o ponto de vista das respectivas idades cronológica dos escolares, presume que o componente genético é o importante fator influenciador no processo de evolução humana ${ }^{1,15,16}$ uma vez que, os escolares com desenvolvimento aparentemente "normal" apresentaram desenvolvimento motor, também normal.

Vale ressaltar que, tal paradigma não constitui uma exarcebação do determinismo genético, sabe-se que outros fatores também podem interferir no processo de desenvolvimento motor e nos resultados obtidos em testes motores específicos, principalmente, as variáveis do ambiente ${ }^{17}$.

Vários autores já estabeleceram relações entre algumas variáveis do ambiente e o desenvolvimento motor de crianças. Ferreira Neto ${ }^{18}$ acredita que, o fato de a criança explorar o ambiente por meio de atividades motoras (como o exercício físico e o jogo) ou pelo desempenho de habilidades motoras implica modificações no seu desenvolvimento físico, perceptivo-motor, como também moral e afetivo. Nesse sentindo, apesar de na atual pesquisa não ter sido feita nenhuma correlação entre desenvolvimento motor e as variáveis do ambiente, pode-se dizer que, nos casos aqui específicos (onde $1 \%$ apresentou superioridade motora e 3\% déficits motores), estes escolares podem estar usufruindo o contexto em que estão inseridos de maneiras desiguais. Vale ressaltar que, o ser humano se desenvolve em diversos ritmos, que dependem de suas próprias características e das experiências vivenciadas, o que possibilita que diferentes padrões de movimento sejam detectados ${ }^{19}$. Não obstante, esses dados vêm apoiar os conceitos de continuidade, individualidade no processo desenvolvimentista ${ }^{1}$.

Especificamente, um dos componentes que merecem atenção nesse estudo é a organização espacial. Apesar de situar-se dentro dos padrões de normalidade, obteve baixo índice, e advém, provavelmente, porque no teste específico dessa área os avaliados precisam de uma boa noção de "direita" e "esquerda". E ficou evidente que muitos escolares apresentaram dificuldades em saber qual é a sua mão direita e esquerda - tarefa exigida no item 6 anos desse teste. Nessa tarefa, todos os escolares da amostra estariam aptos a desempenhar, de acordo 
com a faixa etária que se apresentam. Em um estudo com 207 escolares, a organização espacial foi a área de maior comprometimento motor, e esse baixo escore possa ser decorrentes de uma deficiência na educação brasileira ${ }^{20}$. A estruturação espacial é parte integrante da lateralidade e, por isso, desorientação espacial podem estar relacionadas a presença de lateralidade cruzada ${ }^{21}$. Não obstante, aproximadamente, 1/3 dos escolares da amostra apresentam lateralidade cruzada, apoiando a baixa classificação que obteve a orientação espacial nessa pesquisa.

A determinação da lateralidade se estabelece por volta dos seis anos ${ }^{22}$. Assim sendo, as crianças pertencentes a este estudo encontram-se de acordo com a literatura encontrada, pois a lateralidade na maioria das crianças foi definida. O predomínio foi da preferência lateral direita, concordando com várias pesquisas com escolares nessa faixa etária ${ }^{4,10,20,23}$. Tais índices já eram de se esperar, uma vez que é consenso na população mundial, a prevalência do domínio destro.

Em suma, vale ressaltar que, além das restrições orgânicas e do contexto ou ambiente em que as crianças estão inseridas, as exigências das tarefas propostas são muito relevantes na determinação dos padrões motores ${ }^{23}$. Na tarefa está a maior possibilidade de intervenção do profissional de Educação Física $^{24}$, e para que a intervenção traga resultados positivos é preciso conhecer o indivíduo, finalidade de qualquer avaliação e especialmente neste estudo, a finalidade da avaliação motora proposta.

Alguns estudos interrogam se os profissionais da área da Educação Física avaliam adequadamente o comportamento de seus alunos ${ }^{25}$. Numa pesqui$\mathrm{sa}^{26}$ cujo objetivo foi analisar o conhecimento dos professores de Educação Física acerca dos métodos de avaliação do desenvolvimento motor, foi constatado que apesar de a maioria dos professores considerar importante a realização da avaliação motora, muitos alegaram não realizar. Uma preocupante evidência foi a falta de conhecimento dos professores (quase que na sua totalidade) sobre os instrumentos de avaliação motora existentes, cujas bases são norteadas por normas estabelecidas. Poucos que afirmaram realizar avaliação motora, realizam-na com instrumentos que não são, efetivamente, testes de avaliação motora.

O instrumento para a mensuração do desenvolvimento deve ser coerente com o objetivo que este pretende atingir, e seus resultados devem ser confiáveis e relevantes para que a intervenção seja estruturada ${ }^{27}$. O instrumento utilizado no presente estudo, ao quantificar a Idade Motora e o seu relacionamento com a
Idade Cronológica, comprovou que as tarefas propostas para cada idade na escala, refletem as mudanças esperadas quando ao desenvolvimento motor. Esta correlação, que foi evidenciada no presente estudo, está apoiada na afirmação do autor desta escala, à medida que a criança vai crescendo (aumentando sua idade cronológica) paralelamente o seu nível de desenvolvimento motor também aumenta ${ }^{9}$. Numa pesquisa que analisou a ordenação e os graus de dificuldades das tarefas dos testes motores de equilíbrio acerca da Escala de Desenvolvimento Motor, concluíram que a ordenação propostas para este teste apresenta validade de construção, com incrementos nos graus de complexidade de acordo com a idade cronológica ${ }^{27}$. Num estudo mais recente, que propôs validar as baterias de testes de motricidade global e equilíbrio da Escala de Desenvolvimento Motor, encontrou consistência interna item-escala boa para motricidade global e fraca para o equilíbrio, com alta correlação para a motricidade global e correlação moderada para o equilíbrio, e consistência interna inter-item boa para ambas baterias $^{28}$. Quanto mais altos forem os coeficientes de correlação para validade, fidedignidade e objetividade, sua avaliação terá maior confiabilidade ${ }^{29}$.

Neste estudo, a confiabilidade apontada através da Consistência Interna, que verificou a congruência entre a variável Idade Motora Geral e Idade cronológica, foi elemento essencial para avaliar a qualidade e eficiência deste instrumento de mensuração, contribuindo para a disponibilidade de dados fidedignos relativos a capacidades motoras da população escolar brasileira, visto sua incipiência ${ }^{30}$.

\section{CONCLUSÃO}

De modo geral, o desenvolvimento motor dos escolares encontra-se dentro dos parâmetros de normalidade, evidenciando que o desempenho motor está correlacionado ao desempenho escolar, já que tais escolares não apresentavam queixa de dificuldades na aprendizagem. Além disso, os padrões de movimento obedeceram à sequência das idades cronológica, que através da EDM, refletiu com veracidade as mudanças esperadas quando ao desenvolvimento motor.

A utilização deste instrumento de avaliação oportunizará ao professor a criação de metas educacionais, bem como a identificação de componentes do desenvolvimento motor que necessitam de atenção especial.

\section{REFERÊNCIAS BIBLIOGRÁFICAS}

1. Gallahue DL, Ozmun JC. Compreendendo o desenvolvimento motor: bebês, crianças, adolescentes e adultos. São Paulo: Ed. Phorte; 2005. 
2. Santos S, Dantas L, Oliveira JA. Desenvolvimento motor de crianças, de idosos, e de pessoas com transtorno da coordenação. Rev Paul Educ Fís 2004;18:33-44.

3. Medina J, Rosa GKB, Marques I. Desenvolvimento da organização temporal de crianças com dificuldades de aprendizagem. Rev Educ Fís/UEM 2006;17(1):107-16.

4. Batistella PA. Estudo de parâmetros motores em escolares com idade de 6 a 10 anos na cidade de Cruz Alta - RS. [Dissertação de Mestrado - Programa de pós-graduação em Ciências do Movimento Humano]. Florianópolis (SC): Universidade do Estado de Santa Catarina; 2001.

5. Gregório CSB, Pinheiro ECT, Campos DEO, Alfaro EJ. Evolução neuromotora de um recém-nascido pré-termo e a correção com os fatores perinatais. Fisiot Bras 2002;3(4):250-5.

6. Rosa GKB, Marques I, Papst JM, Gobbi LTB. Desenvolvimento motor de criança com paralisia cerebral: avaliação e intervenção. Rev Bras Educ Esp 2008;14(2):163-76.

7. Brasil. Secretaria de Educação Fundamental. Parâmetros Curriculares Nacionais: Introdução aos Parâmetros Curriculares Nacionais. Brasília: MEC/SEF, 1997.

8. Valentini NC, Barbosa MLL, Cini GV, Pick RK, Spessato BC, Balbinotti MAA. Teste de Desenvolvimento Motor Grosso: Validade e consistência interna para uma população Gaúcha. Rev Bras Cineantropom Desempenho Hum 2008;10(4):399-404.

9. Rosa Neto F. Manual de Avaliação Motora. Porto Alegre: Artmed; 2002.

10. Rosa Neto F. Valoracion del desarrollo motor y su correlacion con los trastornos del aprendizaje[Tese de doutorado]. Zaragoza: Universidade de Zaragoza; 1996.

11. Majorek M, Tuchelmann T, Heusser P. Therapeutic Eurythamy - movement therapy for children with attention déficit hyperactivity disorder (ADHD): a pilot study. Complement Ther Clin Pract 2004;10(1):46-53.

12. Alvim PB, Borges OP. Análise da relação da estrutura espaço-temporal e alfabetização em crianças de 6 e 7 anos. Rev Port Cienc Desp 2004;4(2):155-67.

13. Bradley N. What are the principles of motor development? In: Forssberg H, Hirschfield H.(Eds.), Movement disorders in children. Basel: Karger; 1995. p. 41-49.

14. Ferreira LF, Nascimento RO, Apolinário MR, Freudenheim AM. Desordem da coordenação do desenvolvimento. Rev Motriz 2006;12(3):283-92.

15. Bradley RH, Corwyn RF. Socioeconomic Status and Child Development. Annu RevPsychol 2002;53:371-399.

16. Guedes DP, Guedes JERP. Crescimento composição corporal e desempenho motor de crianças e adolescentes. São Paulo: Balieiro editores; 1997.17. Fonseca FR, Beltrame TS, Tkac CM. Relação entre o nível de desenvolvimento motor e variáveis do contexto de desenvolvimento de crianças. Rev Educ Fis/UEM 2008;19(2): 183-94.

17. Ferreira Neto CA. Desenvolvimento da motricidade e as culturas da infância. In: Moreira WW, organizdor. Educação Física: intervenção e conhecimento científico. Piracicaba: Ed. da UNIMEP, 2004. p. 35-50.

18. Manoel E. Estudo do desenvolvimento motor: Tendências e perspectivas. In: G Tani, organizador. Comportamento motor: Aprendizagem e desenvolvimento. 1 ed. Rio de Janeiro: Guanabara Koogan; 2005.
19. Sabagg S. Percepção dos estereótipos de gênero na avaliação do desenvolvimento motor de meninos e meninas. 2008. [Dissertação de Mestrado - Programa de pós-graduação em Ciências do Movimento Humano]. Florianópolis (SC): Universidade do Estado de Santa Catarina; 2008.

20. Serafin G, Peres LS, Corseuil HX. Lateralidade: conhecimentos básicos e fatores de dominância em escolares de 7 a 10 anos. Caderno de Educação Física 2000;2(1):11-30.

21. Rodrigues LR. Caracterização do desenvolvimento físico, motor e psicossocial de pré-escolares de Florianópolis - SC. [Dissertação de Mestrado - Programa de pós graduação em Ciências do Movimento Humano]. Florianópolis (SC): Universidade do Estado de Santa Catarina; 2000

22. Silveira CRA, Gobbi LTB, Caetano MJD, Rossi ACS, Candido RP. Avaliação motora de pré-escolares: relações entre idade motora e idade cronológica. Lecturas: Educación Fisica y Deportes [Periódico on-line]. 2005.

23. Barela JA. Perspectiva dos sistemas dinâmicos: teoria e aplicação no estudo de desenvolvimento motor. In: Pellegrini AM, organizadora. Coletânea de Estudos: Comportamento Motor I. São Paulo: Movimento;1997. p.11-28.

24. Safrit MJ, Wood TM: Introduction to Mesurement in Physical Education and Exercise Science. St. Louis: Mosby; 1995.

25. Pires DCMF. Avaliação do desenvolvimento motor: uma análise acerca do conhecimento dos professores de educação física. Programa de Desenvolvimento Educacional do Paraná. 2007; Disponível em: <www. pde.pr.gov.br> [2009 out 11].

26. Silveira CRA, Menuchi MRTP, Simões CSA, Caetano MJD, Gobbi LTB. Validade de construção em testes de equilíbrio: ordenação cronológica na apresentação das tarefas. Rev Bras Cineantropom Desempenho Hum 2006;8(3):66-72.

27. Amaro KN, Santos APM, Brusamarello S, Xavier RFC, Rosa Neto F. Validação das baterias de testes de motricidade global e equilíbrio da EDM. Rev Bras Cienc Mov 2009; 17(2)."em editoração".

28. Udinsky BF, Osterlind SJ, Lynch SW. Evaluation resource Handbook: gathering, analyzing, reporting data. San Diego: Edits Publishers; 1981. p. 197-204.

29. Collet C, Folle A, Pelozin F, Botti M, Nascimento JV. Nível de coordenação motora de escolares da rede estadual da cidade de Florianópolis. Rev Motriz 2008; 14 (4):373-380.

\section{Endereço para correspondência}

Francisco Rosa Neto

Universidade do Estado de Santa Catarina, Centro de Ciências da Saúde e do Esporte, Laboratório de Desenvolvimento Humano. Florianópolis, SC. Brasil.

Rua Pascoal Simone, 358 - Coqueiros, CEP 88080-350 - Florianópolis - SC, Brasil. Email: franciscorosaneto@terra.com.br 